\title{
QUALITY \\ IN HEALTH \\ CARE
}

\section{Editorials}

\section{I forgot to ask...}

Simple interventions are sometimes very effective. Providing parents of children attending a paediatric neurology clinic with a sheet inviting written questions encourages many to ask questions that otherwise may not have been articulated - at least not in the clinic. ${ }^{1}$ The immediate benefits to parents, and children, whose worries were resolved are clear, but doctors also noted that parents seemed to take more initiative on subsequent visits. This approach to enabling patients to discuss questions and concerns has wide applicability. It is not a new idea - the authors refer to some of the background literature - and to an outsider it must seem staggering that the use of simple question sheets is not standard clinic practice throughout the NHS.

This story of the introduction of an intervention that does not cost much, has obvious benefit, and probably little potential for harm illustrates two important barriers to quality improvement. Firstly, the information gap: unless we know that there is a better way of doing things then change is unlikely. Secondly, the education gap: unless healthcare professionals have the training that enables them to understand health care from patients' perspective it will be difficult for them to respond effectively to patients' expectations and needs.

Information is one of the major currencies of health care. Patients and healthcare professionals need it, use it, and exchange it. In the past healthcare professionals - especially doctors-had an assumed monopoly on information. But that has changed: technology now allows everyone immediate access to a vast amount of information about health care, biomedical advances, and other innovations in health care. Yet the fact that large amounts of information are available does not mean that people are necessarily more knowledgeable or better equipped to use it.

The key is to encourage the dissemination of effective information - that is, information that seems relevant to the recipients and which they can use. The principles are the same whether the recipients of information are healthcare professionals or their patients. The case for exploring and understanding the dissemination process, finding out what helps, and what is a waste of time, is made in another paper in this issue. ${ }^{2}$ We know, for example, that the provision of information alone is unlikely to be sufficient. Marriott et al show that multiple channels may be needed; care must be taken not to overload the recipients of information; the process of informing may need to be done in stages; and recipients' existing attitudes and beliefs need to be understood.
Moreover, some kinds of information are much more available than others. Healthcare practitioners are increasingly likely to have access to information about clinical interventions, but much less information is available about the merits of organisational interventions or about simple ways of making patients' interactions with healthcare services more tolerable or about how to enable patients to get the advice and reassurance they need from their doctors.

Many patients experience a gap between what they expect and what they get. That some doctors were surprised to find that some of the questions parents wrote on their question sheets were "biologically implausible" is revealing and suggests a gap in medical education. Biomedical science is the basis of many clinical interventions and is the cornerstone of medical education. But effective health care depends on an understanding of many other disciplines. For health care to progress and improve, those working in clinical disciplines need to be provided with the appropriate education and range of skills and competencies to allow them to meet the expectations and needs of their patients. Patients' expectations have changed, so consultations should be less about telling patients what they should do or what will be done and more about exploring and meeting their needs. Doctors should be advisors able to help to steer and address concerns rather than be sole arbiters of what is to be done.

Knowledge alone is not enough. As the pace of change quickens and ever more effective interventions become available, healthcare professionals need the skills to be able to change their own practice and to work with others to make the necessary organisational changes to support improvements in the quality of care. The theoretical background of quality improvement comes from several disciplines, including statistics, social psychology, industrial engineering, and systems theory. ${ }^{3}$ The skills needed include the ability to aggregate outcome data, to understand work as a process, and to enter into collaborative exchange with patients; team working skills ${ }^{4}$; and an understanding of organisational development and change management. ${ }^{5}$ But these are unlikely to feature prominently, if at all, in undergraduate or even postgraduate curricula.

Schon questioned the assumption that professional knowledge taught in schools prepares practitioners for the real world of routine practice. ${ }^{6}$ Reducing this mismatch between what is taught and the skills needed for effective practice should be a matter of priority. Perhaps the first step is to define effective practice in terms of the skills 
needed to meet the expectations and needs of both present and future patients. These skills, will, of course, include technical and clinical competencies and knowledge of biomedical science. But they also include the ability to help patients to express their deep concerns-however implausible - and to be able to come back and say "I forgot to ask..."

FIONA MOSS
Cunningham C, Newton R. A question sheet to encourage written consultation questions. Ouality in Health Care 2000;9:42-6.

Marriott S, Palmer C, Lelliott P. Disseminating healthcare information: getting the message across. Quality in Health Care 2000;9:58-62

3 Berwick D, Enthoven A, Bunker JP. Quality management in the NHS: the doctor's role-1. BMF 1992;304:235-9.

4 Berwick D, Enthoven A, Bunker JP. Quality management in the NHS: the doctor's role-2. BMF 1992;304:304-8.

5 Garside P. Organisational context for quality: lessons from the fields of organisational development and change management. Quality in Health Care 1998; 7(suppl):S8-S15.

6 Schon DA. Educating the reflective practitioner. Toward a new design for teaching and learning in the professions. Oxford: Jossey Bass, 1987.

\section{Improving surgical care: looking beyond individual competence}

In 1998 the General Medical Council (GMC) - the body responsible for registering doctors and for setting standards of professional behaviour in the UK-found three senior doctors from Bristol guilty of professional misconduct. The story, a complex one, centred on the work of two cardiac surgeons whose operative mortality for difficult operations for children with congenital heart disease (transposition of the great arteries and complete atrioventricular septal defects) was higher than expected. ${ }^{1}$ There was public outcry. The surgeons were jostled outside the GMC's building by angry crowds shouting "murdering bastards" and carrying cardboard coffins. $^{2}$

These events-now known simply as "Bristol"-are the subject of a public inquiry looking in depth at the circumstances of the unnecessary deaths (the inquiry can be followed on the Bristol web site http//:www. bristol-inquiry.org.uk). The focus of the GMC was the surgeons' professional conduct. The inquiry, probing into aspects of patient care beyond individual competence, has found that "institutional and organisational problems also played an important part in determining outcome, particularly in higher risk cases". ${ }^{3}$ These issues are likely to have an impact on British medical practice far beyond the detail of complex paediatric cardiac surgery.

Cardiac surgery is only possible with organisational support. In addition to technical competence, successful heart surgery requires appropriate case selection, accurate preoperative diagnosis, detailed preparation for surgery, skilled anaesthesia, and good postoperative care. Coronary artery surgery is the most frequently performed cardiac operation. In the UK each year 25000 operations are done; a median of 800 cases in each of the 35 NHS cardiac units. ${ }^{4}$ Mortality rates provide a means of comparing care among units and among surgeons; figures are available. ${ }^{4}$ Nearly every patient survives. Over $75 \%$ of the 170 cardiac surgeons who do these operations report hospital survival rates of better than $97 \%$, and about $90 \%$ of patients have an uncomplicated recovery and enjoy a good long term outcome. How should surgeons and units react when such high expectations are not fully met? Is the surgeon responsible or is there a problem elsewhere in the team? Or could there be organisational factors in the institution or in the complex chain of case selection and preparation for surgery? If so, what are they likely to be, how do we find out, and how do we improve?

Case selection is the first step. The purpose of cardiac surgery is to reverse or ameliorate problems that are life threatening or cause symptoms which diminish the quality of life. ${ }^{6}$ Benefit must be balanced against risk. All cardiac surgery is risky and some patients face more risk than others. But often it is those with the most critical cardiac disease, for whom perioperative risk is greatest, who have most to gain. The price of longer term benefit may have to be balanced by higher short term risk. A unit setting out to deliver cardiac surgery with the lowest possible perioperative mortality will disadvantage some of those with most to gain. Surgeons taking on higher risk cases will have more deaths. Hospital mortality figures may be wrongly interpreted without a sophisticated understanding of case mix.

All active coronary surgeons operate on a group of patients with more complex conditions than in previous years when the expectations were set by randomised trial. ${ }^{8}$ In the UK, about a quarter of coronary artery surgery is on people admitted with myocardial infarction or for acute angina. About a third are over 70 years of age. ${ }^{5}$ Much case selection is in the hands of referring physicians and investigating cardiologists before the patient sees the surgeon. By the time the patient is in a cardiac centre with an angiogram performed the expectation is that a curative operation will follow. If the operation is not successful, to what extent are referring physicians who force a decision prepared to take responsibility? Sometimes the best the surgeon can do is to ensure that risk stratified data are kept to defend against subsequent criticism of high mortality. ${ }^{7}$

The operation itself is essentially mechanistic. If the coronaries are grafted faultlessly, the patient leaves the operating room with a better blood supply to heart muscle. On the perfect day heart surgery goes like clockwork. These are the days of miracle and wonder, when the heart beats in sinus rhythm, the monitoring lines are placed first time, the electronics work at the flick of a switch, and the physiological variables (circulating volume, left ventricular wall tension, pulmonary and systemic vascular resistance) are controlled to a nicety. The surgeon, working against the clock, sews small grafts to even smaller $(1.5 \mathrm{~mm})$ coronary arteries. There is little margin for error. Unless the patient leaves the operating theatre with a well vascularised myocardium, what are the prospects of withstanding protracted anaesthesia, major surgery, and the effects of cardiopulmonary bypass?

The surgeon's role is crucial. Technical competence is an absolute requirement of the operator; no one else in the cardiac unit can cut and sew the heart. To what extent is the surgeon answerable for the rest? Leadership and decisiveness are also necessary skills for the surgeon. The team expects the surgeon to recognise and diagnose the cause of problems as they present and to solve them, ${ }^{9}$ with the minimum of upset. 
Surgeons cannot be answerable for all mistakes and poor case selection, errors in diagnosis, and poor theatre and postoperative management can be as disastrous as a surgeon's technical lapse Yet poor outcomes, whatever the cause, reflect on the surgeon. As team leaders, surgeons have a special responsibility. And yet unlike leaders in other fields they are unlikely to have had any training in leadership skills. The findings of the Bristol inquiry may be a watershed in our understanding of the complex set of interactions that are needed if the dangers inherent in high risk interventions are to be minimised. Many more questions will be raised than currently we have answers for. And some of the answers will not be in the technical language that surgeons are used to. ${ }^{10}$ By recognising both the technical and organisational aspects of surgery and looking at the performance of the whole, and of teams as well as individuals, we should be able to develop a methodology to enable units and surgeons whose mortality and morbidity figures are worse than expected to diagnose and correct problems in the series of events that make up a surgical procedure. ${ }^{10}$
But perhaps more importantly by understanding the organisational context of surgery we may be better able to manage the system as a whole and reduce the risk of surgery.

TOM TREASURE

Professor in Cardiothoracic Surgery, St George's Hospital, London, UK

1 Treasure T. Lessons from the Bristol case. BMf 1998;316:1685-6.

Dyer C. Compensation claims expected to follow GMC's findings. BMF 1998;316:1691

Dyer C. Study identifies why child heart operations go wrong. BMf 1999;319:803.

4 Society of Cardiothoracic Surgeons of Great Britain and Ireland. UK cardiac surgery register. London: Society of Cardiothoracic Surgeons of Great Britain and Ireland.

5 Keogh BE, Kinsman R. National adult cardiac surgery database report 1998. London: Society of Cardiothoracic Surgeons of Great Britain and Ireland, May 1999.

6 Pocock SJ, Henderson RA, Seed P, et al. Quality of life, employment status and anginal symptoms after coronary angioplasty or bypass surgery: three years follow-up in the randomised intervention treatment of angina (RITA) trial. Circulation 1996;94:135B-2.

7 Treasure T. Risks and results of surgery. Br Heart $\mathcal{f}$ 1995;74:11-12.

8 Kumar P, Treasure T. Coronary artery bypass graft trials. Br f Hosp Med 1996;56:33-6.

9 Treasure T. Cardiac surgery in the Dunkirk spirit. BMF 1995;311:1648. 10 De Leval M. 7 Cardiothor Surg 2000 (in press). 